\title{
Deep learning network based lifetime analysis of energy - fed traction power supply converter
}

\author{
Zeshu Li, Mingchao Xia, and Qifang Chen* \\ School of Electrical Engineering, Beijing Jiao tong University, China
}

\begin{abstract}
This paper presents a life prediction method based on the parameters of the actual operation history data collected by the existing converter power unit sensors. Firstly, the characteristics of junction temperature curves of forced air-cooled radiator and power unit are extracted, and the deep learning neural network architecture is constructed based on the characteristics. Then the thermoelectric coupling model of power unit based on thermal resistance calculation theory is established, and the cumulative loss is obtained from the measured data. The deep learning network is trained and the model prediction is verified. Finally, the power unit loss distribution under different setting temperature thresholds and the correlation analysis with radiator parameters are obtained, which provides a feasible scheme for parameter setting and life prediction.
\end{abstract}

Keywords: Inverter, IGBT, Lifetime prediction, Deep learning.

\section{Energy feed power supply system structure and main research objects}

\subsection{Structure of energy-fed power supply system}

At present, regenerative braking is widely used in urban rail transit vehicles. According to IEC and correlative regulations, the allowable voltage fluctuation range of DC1500V overhead catenary is $1000 \sim 1800 \mathrm{~V}$. The existing power supply system uses diode rectifier power supply, when the regenerative braking energy cannot be fully absorbed by adjacent trains, the grid voltage will rise beyond the limit, leading to the failure of regenerative braking and loss. The main solutions for recycling braking energy are inverter feedback which has the functions of traction power supply, energy feedback and reactive power compensation. The core of the medium voltage inverter feedback device is the three-phase voltage source inverter, which is connected in parallel with the diode rectifier in the traction power supply system. When the train is braking, the excess energy on the DC side is fed back to the medium voltage AC grid [1].

\footnotetext{
*Corresponding author:18121473@bjtu.edu.cn
} 
Each PWM converter unit is composed of several IGBT half bridge modules in parallel. Its DC side is connected in series or parallel according to the capacity requirements, and its $\mathrm{AC}$ side is connected to the transformer after parallel connection. When the train brake and feedback the braking energy to the system, the converter works in the inverter condition to transmit the energy to the medium voltage grid.

\subsection{Influence factors of converter lifetime}

Studies have shown that power devices and electrolytic capacitors are the highest rate of fault aging, and they are also the most important components that determine the life of the entire equipment. This paper mainly analyses and studies the power devices and related equipment of the converter.

For IGBT, the aging failure of bonding wire can be evaluated by saturated conduction pressure drop $\left(\mathrm{V}_{\text {cesat }}\right)$, and the fatigue aging of weld layer fatigue is positively correlated with the junction-case thermal resistance $\left(\mathrm{T}_{\mathrm{j}-\mathrm{c}}\right)[2]$.

In general, the two methods are material fatigue caused by thermal stress, and the temperature curve of power units is essentially determined by the heating process caused by transmission power loss and heat dissipation process cooling caused by system operation.

\section{Forced air-cooling and characteristics of historical data}

Heat sink is one of the main component of converter heat dissipation structures, and forced air cooling is often used for high-power converter in urban rail transit. The chip temperature is detected by the substrate temperature sensor placed on the chip's substrate.

The fluctuation curve of the junction temperature $\left(T_{j}\right)$ is affected not only by the working condition of the chip and the external temperature, but also affected by the working condition of the cooling system. Without starting the cooling system, when the power module is working on inverter state, the energy is sent back to the AC side medium voltage power grid from the DC side feeder through the IGBT chip and diode, resulting in loss and heat on the chip, and the junction temperature and measurement temperature rise. When the energy return process is finished, the chip is cooled slightly and maintained at a certain temperature higher than the increased environment. During the operation of the power supply system, these two conditions alternate, and making the mean value of junction temperature get higher. On this basis, the temperature peak value of the energy feedback process is also increased. When the power unit is detected to exceed the set temperature threshold, the forced air-cooling process is started for rapid cooling. When the detected temperature value is lower than the lower limit of the set temperature threshold and the thermal stability is maintained, the cooling fan is turned off.

Therefore, with the participation of the cooling process of forced air-cooling system, the temperature measured by power module is in a cycle process of alternating heating and cooling, and the fluctuation range of is determined by the upper and lower threshold temperature of the cooling system, and the taken time for a cycle process is often positively related to the transmission power in this period. Therefore, based on the historical temperature, AC / DC voltage and current data of the converter power module detected by the existing sensors of converter power units, this paper studies the lifetime of the power unit under different temperature thresholds and the correlation analysis with the radiator parameters. 


\section{Data set and deep neural network establishment}

In this paper, the deep learning network is used to fit and predict the historical data, and the data set is formed by feature extraction of the historical data. The deep learning network is trained and verified.

As described in the previous chapter, the measured temperature is closely related to the working condition of the power units and the parameters of the cooling system. Due to the high correlation between electrical quantities, the transmission power is analyzed as its replacement in this paper. Most of the parameters of the cooling system are fixed, and as mentioned above, the most important parameter that affect the operation of the cooling system is the start-up temperature threshold. In order to study the influence of different start-up thresholds on the system, this paper takes them as the main research object.

Because of the periodic cycle characteristics of temperature fluctuation series data, the current value is related to the fluctuation of past time, and the data amount is large. Considering these factors, each heating process and cooling process are segmented when extracting data characteristics, and a certain temperature interval is set to divide each segment, from the beginning of the process or the end of the cooling process to the temperature interval. The data feature is extracted by polynomial fitting. Under this condition, the polynomial can meet the accuracy requirements by taking the fourth or fifth order.

For the structure design of deep learning artificial neural network, according to the literature and the fitting results of the specific model data, the preliminary parameter adjustment was carried out, the parameters of the neural network are shown in Table 1.

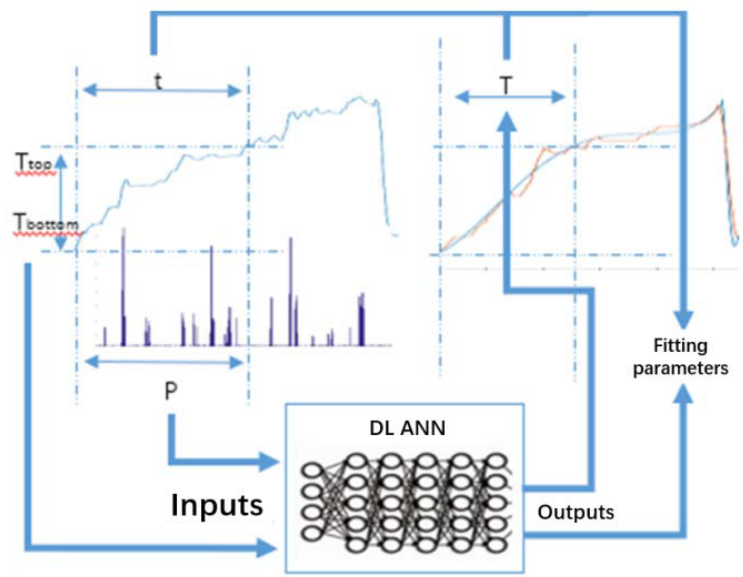

Fig. 1. Working process of deep neural network.

Table 1. DL ANN parameters

\begin{tabular}{|c|c|}
\hline Number of layers & 5 \\
\hline Number of units in each layer & $(12,50,200,200,6)$ \\
\hline training method & SCG \\
\hline Learning rate & 0.005 \\
\hline
\end{tabular}

The transmission power in the predicted time range and the upper and lower extreme values of the temperature in this section are normalized to form the input layer, and the output layer is used to fit the fourth-order polynomial parameters of the temperature fluctuation curve and predict the process time. 
Taking the prediction of heating process as an example, as shown in Figure 1, for the prediction process time obtained by the output layer and the selected input time, when the error exceeds the limit, the input time is corresponding shortened or extended to adjust the error. Then, a new training or prediction is carried out from the next period of time until the extremum of the output curve reaches the given working threshold of the controlled cooling system. In the same way, the prediction process of the temperature fluctuation curve under the working condition of the cooling system is carried out, so as to obtain the overall temperature change prediction curve.

\section{Calculation of average loss of power unit and junction temperature}

\subsection{Establishment of thermoelectric coupling model}

The total power loss of the half bridge module used in the power unit is mainly composed of on-state loss and switching loss of IGBT and its antiparallel freewheeling diode (FWD). The switching loss is composed of turn-on loss and turn-off loss. The power loss of IGBT is composed of two parts, namely, the $P_{\text {cond_Q } 1}$ in the turn-on process, and $\mathrm{P}_{\mathrm{Sw}_{-} \mathrm{Q} 1}$ during switching, the calculation process is as follows:

$$
\begin{gathered}
P_{\text {cond_Q } Q 1}=\frac{1}{2 \pi} \sum_{k=1}^{4} \int_{\alpha_{k}}^{\beta_{k}} v_{c e}\left(t, T_{j}\right) \times I_{c}(t) \times \delta_{k} d \omega t \\
P_{S w_{Q 1}}=f_{s w} \times \frac{1}{2 \pi}\left(E_{o n}\left(t, T_{j}\right)+E_{o f f}\left(t, T_{j}\right)\right) \frac{U_{d c}}{U_{n o m}}
\end{gathered}
$$

The power loss of FWD consists of two parts, which are the loss $P_{\text {cond_D4 }}$ in the conduction process, $\mathrm{P}_{\mathrm{Sw} \_\mathrm{D} 4}$ during switching, the calculation process is as follows:

$$
\begin{gathered}
P_{\text {cond }_{D 4}}=\frac{1}{2 \pi} \sum_{k=1}^{4} \int_{\alpha_{k}}^{\beta_{k}} v_{f}\left(t, T_{d}\right) \times I_{f}(t) \times\left(1-\delta_{k}\right) d \omega t \\
P_{s w_{-} Q 1}=f_{s w} \times \frac{1}{\pi} E_{r r}\left(I_{f}, T_{d}\right) \frac{U_{d c}}{U_{n o m}}
\end{gathered}
$$

where: $T_{j}$ is the junction temperature of IGBT; $T_{d}$ is the junction temperature of FWD; $\omega$ is the angular frequency; $f_{s w}$ is the switching frequency; $\delta_{k}$ is the duty ratio of each sector; $\alpha_{k}$ is the starting angle of each sector; $\beta_{k}$ is the termination angle of each sector; $E_{o n}$ is the IGBT switching on power loss; $E_{o f f}$ is the IGBT switching off power loss; $E_{r r}$ is FWD switching off power loss; $I_{c}$ and $I_{f}$ is the on state current of IGBT and FWD; $V_{c e}$ and $V_{f}$ is the conduction voltage drop of IGBT and FWD; $U_{d c}$ is DC bus voltage and $U_{n o m}$ is the rated DC bus voltage.

Due to the structural symmetry of the converter, the multiple control method of the parallel half bridge module of each phase power unit and the current and voltage sharing method, it is only necessary to calculate the power loss of the switches (Q1, D4) with positive A-arm current, and then multiply by the number of switches $n$ to get the power loss of the whole power unit as blow:

$$
P_{\text {per }}=n \times\left(P_{\text {cond_Q1 }}+P_{S w_{-} Q 1}+P_{\text {cond_D4 }}+P_{S w_{-} Q 1}\right)
$$




\subsection{Prediction of $T_{j}$ method}

In this paper, through the thermal network model of IGBT and FWD, the junction temperature of the chip is predicted by the theory of electrothermal coupling. Generally, the fourth-order series parallel RC network can achieve a very high approximation effect. The thermal equivalent circuit is shown in Figure 2, the power loss $P_{\operatorname{tot}(\mathrm{T} / \mathrm{D})}$ is equivalent to a corresponding current source, and the thermal impedance $\mathrm{Z}_{\mathrm{th}(\mathrm{x}-\mathrm{y})}$ is represented by a $\mathrm{RC}$ network.

For the fourth-order thermal impedance RC thermal impedance, the fitting formula is as follows:

$$
Z_{j-c}(t)=\sum_{i=1}^{4} R_{i}\left(1-e^{\frac{-t}{R_{i} C_{i}}}\right)
$$

where: $\mathrm{Z}_{\mathrm{j}-\mathrm{c}}(\mathrm{t})$ represents the junction-case thermal impedance; $R_{i}$ is the thermal resistance; $C_{i}$ represents the heat capacity.

The thermal network Simulink model of junction temperature prediction for IGBT and FWD is shown in Figure 3.

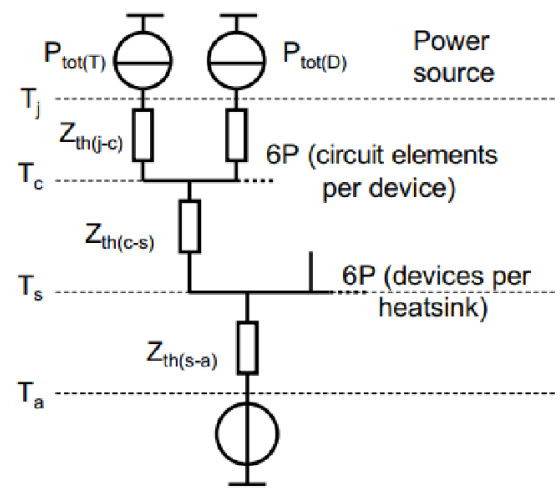

Fig. 2. Simplified thermal equivalent circuit of IGBT and FWD.

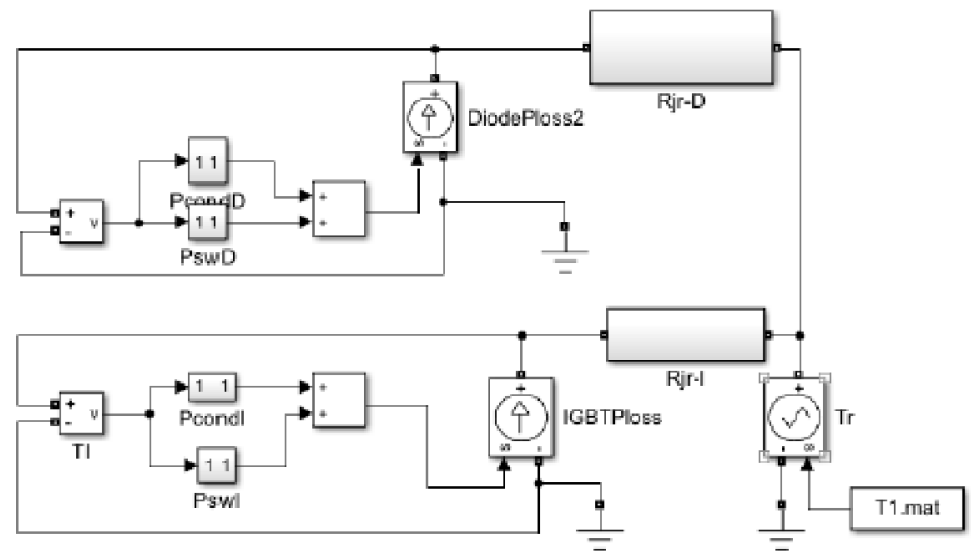

Fig. 3. Simulation model of chip thermoelectric coupling based on Simulink. 
According to the model of power device shown in the Figure 3, the junction temperature calculation formula of the chip is as follows:

$$
\begin{aligned}
& T_{j}(t)=P_{I G B T}(t) \times R_{D j-r}(t)+T_{h s}(t)=\left(P_{P c o n d D}(t)+P_{P s w D}(t)\right) \times R_{D j-r}(t) \\
& T_{j}(t)=P_{I G B T}(t) \times R_{D j-r}(t)+T_{h s}(t)=\left(P_{P c o n d D}(t)+P_{P s w D}(t)\right) \times R_{D j-r}(t)
\end{aligned}
$$

where: $T_{j}(t)$ and $T_{d}(t)$ is the temperature of IGBT and FWD; $R_{j-r}(t), R_{j-r}(t)$ is the fourthorder fitting thermal impedance of IGBT and FWD respectively; $\mathrm{P}_{\mathrm{IGBT}}(\mathrm{t})$ and $\mathrm{P}_{\text {diode }}(\mathrm{t})$ represents IGBT and FWD power loss.

The temperature of the reference point of the power unit temperature can be acquired in real time. Through the above electrothermal coupling calculation model, the junction temperature of the power device switch can be calculated. Then the calculated power loss value is feedback into the electrothermal coupling calculation model, and the junction temperature value at the next moment is obtained by iteration.

We refer to the lifetime modules in reference and datasheet [3][4], using Rain flow counting method to estimate the life damage caused by junction temperature fluctuation.

\section{Training verification and error analysis}

In this paper, the data of energy fed traction power supply station equipment obtained from the actual measurement of Nanning Metro Line 1 in China is used for training. After the above data processing and model parameter adjustment, the training results meet the expectations. It can be seen from Figure 4 that the training effect achieves the accuracy and has a certain degree of generalization, while the value of figure 5 shows the value of $\mathrm{R}$ close to 1 represents that the input and output have strong correlation, and the model parameter adjustment is suitable, these results show that the prediction model is effective.

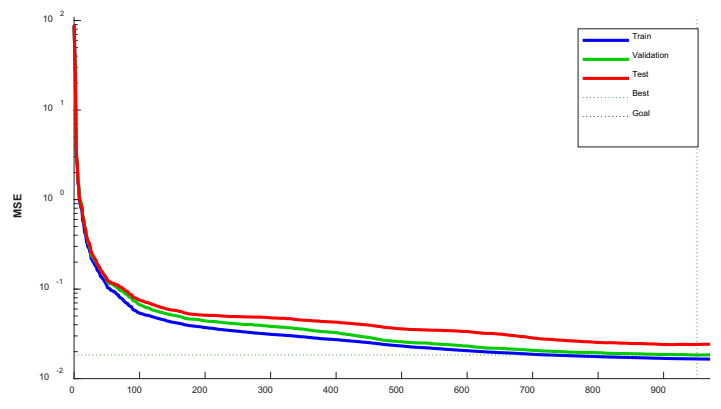

Fig. 4. Training verification results.

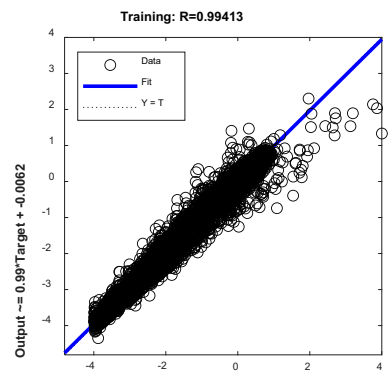

Fig. 5. Training relevance. 
Several classic days outside of the training data set are selected as the prediction objects, and the working threshold of the prediction cooling equipment is set to be the same as the actual situation. The comparison between the neural network prediction and the actual measured value in one day is obtained, as shown in Figure 6. It can be seen that the temperature fluctuation of the device can be well predicted in the rising and falling stages of the temperature, and the main error is as the cumulative error of time prediction in the later period, the total loss error is less than 3\% under the same life model by comparing several classical days and the average life damage obtained by rain flow counting method is also consistent with the expectation.

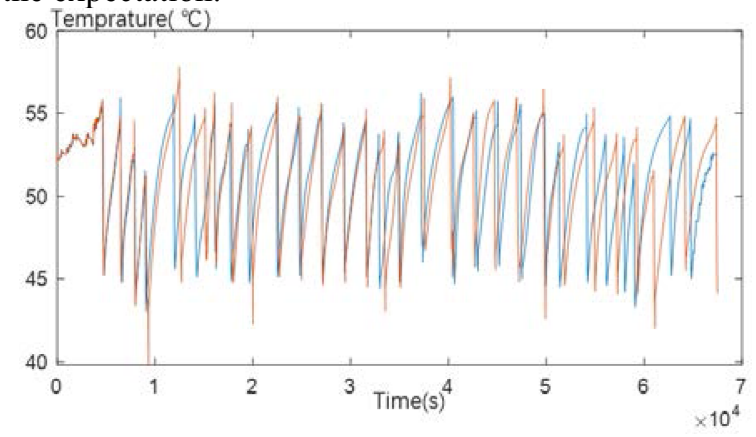

Fig. 6. Comparison of fluctuation of chip measurement value and prediction curve.

When the start-up value of the cooling system is changed, the neural network can predict the temperature cycle curve under the same working condition (i.e., keeping the working condition unchanged), so as to obtain the relationship between this two. As shown in Figure 7, it is the forecast fluctuation curve of the original set starting value and the fourdegree reduced starting value under the same day working condition.

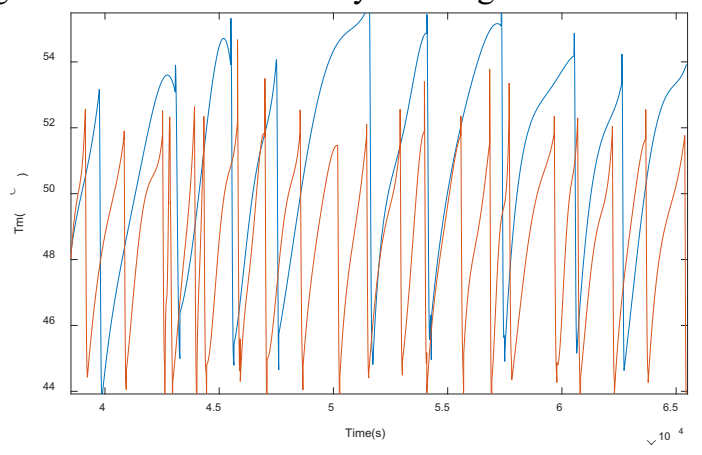

Fig. 7. Temperature curve under different starting thresholds.

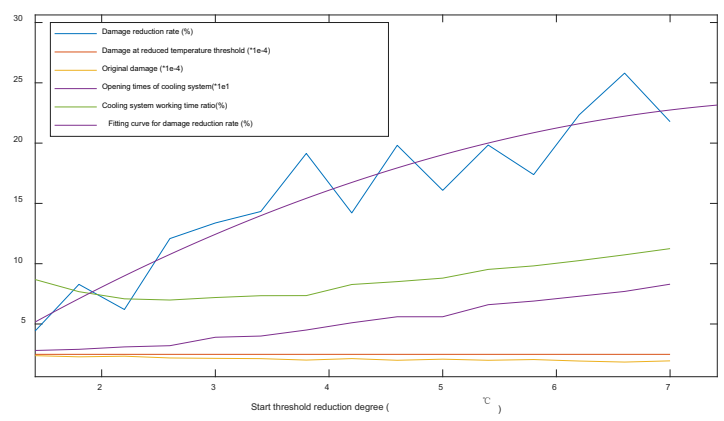

Fig. 8. Changes of system parameters with different starting thresholds. 
It can be seen that the temperature peak value is significantly reduced, but the corresponding temperature rise and fall process times are increased. Changing the system parameters under more start-up values can get the situation as shown in Figure 8 . The results show that although reducing the threshold value can reduce the loss, the maximum amplitude can reach about $20 \%$, but at the same time, the working times and duration of the cooling system will also increase rapidly.

\section{Conclusion}

The thermal parameters of power devices are usually calculated and configured under the premise of maximum junction temperature, without considering the demand and life related factors in practical application, which is not conducive to the coordination and life prediction between the two [5].

In this paper, the temperature change of inverter chip in energy fed traction power supply station is predicted by deep neural network, and the relationship with the working state of cooling system is explored. The results show that there is still room for adjusting the current temperature setting value, but lowering the starting value may not have a positive effect on the overall life of the whole system, so it needs joint analysis to select the appropriate starting value One step to reduce losses and extend life.

\section{References}

1. Zhang G. (2010). Study on Key Techniques of Energy Fed Traction Power System for Urban Mass Transit. (Doctoral dissertation, Beijing Jiao tong University).

2. Vasudevan, V. , \& Fan, X. . (2008). An acceleration model for lead-free (SAC) solder joint reliability under thermal cycling. Electronic Components and Technology Conference, 2008. ECTC 2008. 58th. IEEE.

3. C. Göbl, \& Faltenbacher, J. . (2011). Low temperature sinter technology die attachment for power electronic applications. 2010 6th International Conference on Integrated Power Electronics Systems. IEEE.

4. Yutong Lu, \& Gang Zhang, J. .(2015). Control Method and Application of Energy-Fed Traction Power Supply Device. International Conference on Electrical and Information Technologies for Rail Transportation.

5. Yi, Z. , Wang, H. , Wang, Z., Yang, Y., \& Blaabjerg, F. . (2017). Impact of lifetime model selections on the reliability prediction of IGBT modules in modular multilevel converters. 2017 IEEE Energy Conversion Congress and Exposition (ECCE). IEEE. 